\title{
Metric gravity theories and cosmology. I. Physical interpretation and viability
}

\author{
Leszek M. SOKOŁOWSKI \\ Astronomical Observatory and Centre for Astrophysics, \\ Jagellonian University, \\ Orla 171, Kraków 30-244, Poland \\ uflsokol@th.if.uj.edu.pl
}

Short title: Nonlinear gravity in cosmology

\begin{abstract}
We critically review some concepts underlying current applications of gravity theories with Lagrangians $L=f\left(g_{\mu \nu}, R_{\alpha \beta \mu \nu}\right)$ to cosmology to account for the accelerated expansion of the universe. We argue that one cannot reconstruct the function $f$ from astronomical observations either in cosmology or in the solar system. The Robertson-Walker spacetime is so simple and "flexible" that any cosmic evolution may be fitted by infinite number of various Lagrangians. We show on the example of Newton's gravity that one cannot recover the correct equation of motion from its approximate solution. Any gravity theory different from general relativity generates a new cosmological theory and all the successes of the standard cosmological model are lost even if a single solution of the theory well fits the observations. Prior to application of a given gravity theory to cosmology or elsewhere it is necessary to establish its physical contents and viability. This study may be performed by a universal method of Legendre transforming the initial Lagrangian in a Helmholtz Lagrangian. In this formalism Lagrange equations of motion are of second order and are the Einstein field equations with additional massive spin-zero and spin-two fields. All the gravity theories differ only by a form of interaction terms of the two fields and the metric. Initial conditions for the two fields in the gravitational triplet depend on which frame (i.e., the set of dynamical variables) is physical (i.e. matter is minimally coupled in it). This fact and the multiplicity of possible frames obstruct confrontation of solutions to equations of motion with the observational data. A fundamental and easily applicable criterion of viability of any gravity theory is the existence of a stable ground state solution being either Minkowski, de Sitter or anti-de Sitter space. Stability of the ground state is independent of which frame is physical.
\end{abstract}


PACS numbers: $04.50 .+\mathrm{h}, 98.80 . \mathrm{Jk}$

\section{Introduction: problems with gravity theories}

Metric theories of gravity, where the Lagrangian is any smooth scalar function of the curvature tensor, $L=f\left(g_{\mu \nu}, R_{\alpha \beta \mu \nu}\right)$, named metric nonlinear gravity (NLG) theories, have first attracted attention as possible candidates for foundations of quantum gravity due to their renormalizability properties [1] and then as a possible source of inflationary evolution of the very early universe [2]. A recent revival of interest in these theories has come from cosmology. In fact, the theoretical state of affairs in cosmology is astonishing. The universe consists of baryons (4 percent), unknown stable massive particles forming nonbaryonic dark matter which do not fit the standard model of particle physics (26 percent) and dark energy (70 percent) about which we have only negative knowledge: these are not particles. About 96 percent of the material content of the universe is a great mysterious puzzle.

On the other hand the laboratory experiments and astronomical observations confirming general relativity are still not very numerous and belong to a rather narrow class of tests. It is therefore attractive to conjecture that both the gravitational stability of galaxy clusters and the acceleration of the universe are not due to some unknown forms of matter, but can be accounted for by some modification of gravity theory. Modifications may go in all possible directions; here we deal with the most popular concept, the metric NLG theories. Among these the restricted NLG theories, wherein the Lagrangian is a function of the curvature scalar alone, $L=f(R)$, have been most frequently investigated. This approach to the dark matter and dark energy problem is sometimes referred to as a "curvature quintessence scenario". A typical motivation underlying this approach is following. Consider a Lagrangian of the form $L=R+R^{2}+\frac{1}{R}$ and the Robertson-Walker $(\mathrm{R}-\mathrm{W})$ spacetime. In the very early universe, when the curvature was large, the $R^{2}$ term was dominating generating some kind of inflation [2]. At present $R$ is small and the $\frac{1}{R}$ term dominates giving hopefully rise to the accelerated evolution. And for the most time in the history the curvature scalar had intermediate values so that the linear term was leading preserving all the successes of the standard Friedmann cosmology [3, 4, 5, 6, 7, 8, 9]. This argument, attractive as it sounds, is misleading for three reasons.

Firstly, if one makes a correction to the Einstein-Hilbert Lagrangian in the form $L=R+\varepsilon(R)$ where $\varepsilon(R)$ is any nonlinear function, it is not true that the resulting corrections to solutions of Einstein's theory are small when $\varepsilon(R)$ is very small and become significant only when $\varepsilon(R)$ is sufficiently large. The point is that any nonlinear correction to $L=R$ drastically alters the dynamical structure of the theory: the field equations become of fourth-order instead of second order and the higher curvature terms, even seemingly small, are always very important. To show the effect we consider 
a very simple model, a one-dimensional harmonic oscillator perturbed by introducing a small term with the third derivative,

$$
\varepsilon \dddot{x}+\ddot{x}+\omega^{2} x=0,
$$

with $|\varepsilon| \ll 1$. One seeks for solutions of the form $x=e^{\lambda t}$, then $\lambda=$ const is a solution of a cubic equation $\varepsilon \lambda^{3}+\lambda^{2}+\omega^{2}=0$. One solves it by perturbing the two unperturbed solutions, i.e. one sets $\lambda_{ \pm}= \pm i \omega+\varepsilon \alpha_{ \pm}$. Up to terms linear in $\varepsilon$ the solutions of the cubic equation are $\alpha_{ \pm}=\frac{\omega^{2}}{2}$. Thus one has two almost periodic solutions

$$
x_{ \pm}(t)=a_{ \pm} \exp \left[\left( \pm i \omega+\frac{\omega^{2}}{2} \varepsilon\right) t\right]
$$

being slowly damped or amplified periodic solutions for the unperturbed oscillator. These are approximations to exact solutions which are analytic in $\varepsilon$ at $\varepsilon=0$. However there is also a non-analytic at $\varepsilon=0$ solution of the differential equation corresponding to a third root of the cubic equation. Assuming that $\lambda_{3}$ is of order $\varepsilon^{-1}$ and keeping only the leading terms (of order $\varepsilon^{-2}$ ) one gets $\lambda_{3}=-\frac{1}{\varepsilon}$ and the third solution is exponentially growing $(\varepsilon<0)$ or fading,

$$
x_{3}=a_{3} \exp \left(-\frac{t}{\varepsilon}\right) .
$$

This solution is qualitatively different from the other two and exists for arbitrarily small $\varepsilon$. One expects that the space of solutions is significantly extended by qualitatively new solutions due to any correction $\varepsilon(R)$ to $L=R$.

Secondly, the Lagrangian is not a physical observable whose value or variability gives some insight into the state of a given physical system. It is a kind of a generating function giving rise to equations of motion for the system and observables such as energy-momentum tensors (canonical and variational). Any gravitational Lagrangian is, by definition, made up of scalars, in general these are all invariants of the Riemann tensor (and possibly their derivatives), while the resulting field equations are tensor ones. Any assumption about the value of a scalar appearing in $L$, say $R$, actually tells very little about corresponding solutions. For instance, setting $R=0$ in general relativity one gets not only all vacuum solutions but also those for matter with a traceless energy-momentum tensor. Actually one can say something nontrivial about the sought for solution merely by inspection of the Lagrangian $L=f(R)$ only in the case of the simplest non-maximally symmetric spacetime, the Robertson-Walker one. In fact, the Riemann tensor for this metric is determined by the cosmic scale factor $a(t)$ and for any gravitational Lagrangian (also that explicitly depending on the Weyl curvature) the field equations reduce to one quasilinear third order ODE for $a(t)$, hereafter named the quasi-Friedmannian equation. Then assuming that $R$ is large in some epoch of cosmic evolution and small in another one, one may neglect small terms in this equation and find approximate (or even exact) solutions in these epochs. In so doing one must take 
care since there is no easily available information about values of $R$ in various eras of the cosmic evolution. In fact, in general relativity $R=-8 \pi G T$ where $T$ is the trace of the matter energy-momentum tensor and for cosmic perfect fluid $T=3 p-\rho$. In the early universe the ultrarelativistic plasma has the equation of state $p=\frac{1}{3} \rho$ and then $R=0$. Hence in the transition from the radiation to the matter era $R$ increases, contrary to what has usually been assumed in the motivation for $L=R+R^{2}+\frac{1}{R}$ and only later on it slowly fades. What is more important, the relationship between $R$ and $T$ (as well as between $R_{\mu \nu}$ and $T_{\mu \nu}$ ) is algebraic only in general relativity and for a nonlinear $L=f(R)$ it becomes a differential equation for $R$. If the field equations (in Jordan frame) are $E_{\mu \nu}(g)=8 \pi G T_{\mu \nu}$, then their trace reads

$$
R f^{\prime}(R)-2 f(R)+3 f^{\prime \prime \prime}(R) g^{\mu \nu} R_{, \mu} R_{, \nu}+3 f^{\prime \prime}(R) \square R=8 \pi G T
$$

and one cannot a priori estimate which terms in the equation are dominant and which ones may be neglected. To this end one must assume not only an approximate value of $R$ in a given epoch but also that $R$ is slowly and monotonically varying in order to estimate the derivatives; in this way one eliminates possible rapidly oscillating solutions. All this makes sense if one is convinced that he deals with the correct Lagrangian, e.g. the Lagrangian has been derived from first principles (string theory, quantum gravity etc.) or otherwise motivated. Going in the opposite direction, i.e. attempting, as is recently done, to reconstruct the underlying Lagrangian from observed qualitative features of the cosmic scale factor means that one has to construct the whole relativistic cosmology anew.

In fact, from the general Hawking-Penrose singularity theorem, valid in general relativity, it follows that our universe contains a singularity since the cosmic fluid satisfies the strong energy condition. Then in the case of the Robertson-Walker spacetime the Friedmann equation implies that the singularity was in the past, the initial Big Bang, and the cosmic scale factor monotonically grows from zero at the curvature singularity. We stress that these are generic qualitative properties of any solution to the Friedmann equation, independent of a specific equation of state for the cosmic fluid matter. On the other hand for a generic $L=f(R)$ the singularity theorem cannot hold. Whether or not the theorem holds must be proved case by case 1 . For an arbitrary function $f(R)$, even for many of those Lagrangians which admit a solution qualitatively fitting the astronomical data (an acceleration phase at present preceded by a deceleration phase), there was no initial singularity. There was no Big Bang and the following "early universe" when it was small, dense and hot. And the quasi-Friedmannian equation for such a Lagrangian does not ensure that the cosmic scale factor grows monotonically from an initial small value. What is actually done by those authors who say "today the $R^{-1}$ term

\footnotetext{
${ }^{1}$ For theories in which the theorem holds it takes a form different from that in general relativity since $R_{\mu \nu}$ is not a function of $T_{\mu \nu}$.
} 
is leading in $L$ while in the early universe the $R^{2}$ term was dominant" is merely choosing a class of (approximate) solutions for which $a$ and $R$ significantly and monotonically vary in the course of a cosmic evolution; the solutions in this class are, by construction, ever increasing. This is a kind of "fine tuning" since one may expect that there are classes of qualitatively different solutions which are no less typical. This conjecture is at least partially supported by rigorous investigations in the Einstein frame where the third gravitational degree of freedom for $L=f(R)$ gravity is revealed as a nonlinear scalar field minimally coupled to Einstein gravity: for certain simple (power-law) scalar field potentials all solutions (in the spatially flat Robertson-Walker spacetime) are oscillatory at late times while for potentials which are bounded from above there exist solutions which are global to the past with the Hubble parameter $H=\dot{a} / a$ converging to a constant nonzero value as $t \rightarrow-\infty$ [10]. Taking into account the complexity of the quasi-Friedmannian equation for any nonlinear $f(R)$, investigation of the qualitative properties of all solutions is not easy and can only be done (case by case) by performing a phase-space analysis. Is is fortunate that the equation can be reduced to a first order equation for $\dot{H}$ viewed as a function of $H$ in the case of the Einstein-de Sitter spacetime $(k=0)$ and a second order one for the open and closed universe ${ }^{2}$ (Starobinsky in ref. [2]). A preliminary analysis of evolution of the Einstein-de Sitter metric in the case od a couple of special Lagrangians was performed by Carroll et al. 44. For the Lagrangian most frequently studied, introduced in [3], they found an attractor solution $a \rightarrow t^{2}$ for late times and an exact solution $a \propto t^{1 / 2}$ starting from a curvature singularity at $t=0$. However it is difficult to see from their phase portraits, which cover only a piece of one quadrant of the phase space, whether all solutions emerge from the singularity or there are nonsingular solutions (besides the exponentially growing and decreasing ones with constant $H$ ) and whether oscillatory (non-monotonic) solutions are excluded. It should be emphasized that if for a given accepted Lagrangian the two questions are answered "no", then all the successes of the standard Friedmann cosmology are lost.

The last statement leads us to the third reason, taking a form of a problem: to what extent can one reconstruct the Lagrangian from a given solution? Clearly any given function may be viewed as a solution of many diverse differential equations. Requirement that the function is a solution of a Lagrange equation imposes stringent restrictions on possible equations and allows one to look for a unique answer. A simple example shows that there are cases when it can be effectively done under some conditions. The Newton's gravitational force may be expressed as the gradient of a potential and one seeks for a corresponding Lagrangian. The potential generates a differential scalar $S=\partial_{i} \phi \partial_{i} \phi\left(x_{i}\right.$ are the Cartesian coordinates) and the kinetic part of any Lagrangian should be some function $f(S)$. Furthermore there may be a potential energy $V(\phi)$ and a candidate

\footnotetext{
${ }^{2}$ There is a subtle mathematical assumption necessary to decrease the order of the equation, namely that $H(t)$ is a monotonic function. Then the analysis loses all solutions $a(t)$ which are not monotonic.
} 
Lagrangian is $L=f(S)-V(\phi)$. Inserting the Newton's potential $\phi=-\frac{\alpha}{r}$ into the Lagrange equation

$$
4 f^{\prime \prime}(S) \phi_{, i k} \phi_{, i} \phi_{, k}+2 f^{\prime}(S) \triangle \phi+V^{\prime}(\phi)=0
$$

$\left(\phi_{, i} \equiv \partial_{i} \phi\right)$ one gets

$$
-\frac{8 \alpha^{3}}{r^{7}} f^{\prime \prime}(S)+V^{\prime}(\phi)=0
$$

The problem is indeterminate since there are two unknown functions and one equation. A unique solution corresponding to the Laplace equation arises either upon setting $V=0$ or requiring linearity of the resulting equation.

Suppose now one is attempting to determine the Lagrangian of an underlying restricted NLG theory from observations in the solar system. It is reasonable to assume that the metric is static spherically symmetric. As we discuss further in this work one of essential requirements that any viable $L=f(R)$ theory should satisfy is that it admits solutions either with $R_{\mu \nu}=0$ or $R_{\mu \nu}=\Lambda g_{\mu \nu}$ with $\Lambda$ constant positive or negative (Einstein spaces); clearly there are infinitely many Lagrangians having this feature. Thus each $L=f(R)$ theory under consideration has either Schwarzschild or Schwarzschild-(anti)de Sitter spacetime as a solution and if it is this solution that is realized in the nature then no set of observations and experiments can reconstruct the function $f(R)$. In these theories the Birkhoff theorem does not hold and other static spherically symmetric solutions do exist (very few of them are known [11]). Any solution different from $R_{\mu \nu}=\Lambda g_{\mu \nu}$ is generated by nonzero values of a scalar field being a nongeometric component of a gravitational doublet of fields, see section 3. There are two possibilities depending on whether the doublet is in the Jordan frame (more precisely, in Helmholtz-Jordan frame) or in the Einstein frame. If the measurable quantities form the Jordan frame, then the spin-0 gravity has ordinary matter as a source. In the solar system the source (the sun) enforces spherical symmetry of the scalar. The field is massive and unless its mass is extremely small it is a short range one. The presence of the scalar gives rise to a nonSchwarzschildean solution which is very close to ordinary Schwarzschild one (possibly with $\Lambda \neq 0$ ). Nevertheless a recently found approximate solution for a specific form of $f(R)$ shows that even small corrections are detectabl 3 and in this case they are ruled out by measurements [12. In the case the Einstein frame is observable the scalar is independent of any local matter distribution and in particular in the solar system it is not determined by position and mass of the sun. It is rather of cosmological origin, i.e. the initial data are fixed and common for the entire universe. Then the scalar field is (almost) homogeneous and to avoid a conflict with the cosmological observations it must be very weak giving rise to unmeasurable effects in the solar system. This means

\footnotetext{
${ }^{3}$ This solution belongs to the class of solutions which are generated by the higher-than-second order of the field equations in JF, i.e. it is analogous to the third solution for the perturbed harmonic oscillator discussed above. The solution does not reduce to a Schwarzschildean one in the limit $f(R) \rightarrow R$ and this is why it considerably deviates from the former even if the difference $f(R)-R$ is negligibly small.
} 
that all observations performed there will confirm the Schwarzschild metric. Even the cosmological constant is undetectable locally since the most sensitive to it effect, the perihelion shift of Mercury, requires $|\Lambda| \geq 10^{-41} \mathrm{~m}^{-2}$ [13], while the cosmologically admissible value is $|\Lambda| \approx 10^{-52} \mathrm{~m}^{-2}$. Thus observations may effectively distinguish between the two frames. If Jordan frame is physical then small corrections to Schwarzschild metric generated by the scalar are ruled out by the observations in vicinity of the sun in the case of most Lagrangians investigated up to now [14, 7, 15, 9, 16, 17, 18, 19], though there are arguments that the linear approximation applied to calculate these corrections may not be valid within the solar system [20].

The Robertson-Walker spacetime has a higher (six-dimensional) symmetry group and its metric contains an arbitrary function which may be freely adjusted so that each metric NLG theory, i.e. any $L=f\left(g_{\mu \nu}, R_{\alpha \beta \mu \nu}\right)$, admits it as a solution of the corresponding quasi-Friedmannian equation and in this sense this spacetime is "flexible" and universal while Minkowski, de Sitter and anti-de Sitter spaces are not. Hence one expects that infinitely many of the theories predict a deceleration phase in the past and an accelerated evolution in the present epoch. It has been conjectured that any cosmological evolution may be realized by some specific $L=f(R)$ [6]. And it is impressive that the astronomical data may be fitted by such diverse functions as rational and exponential ones [21], a combination of two confluent hypergeometric functions [6], a combination of two hypergeometric functions [22] and even an implicit form of $f(R)$ was found which is consistent with the three years collection of WMAP data [6]. Also a "toy model" based on a minimal curvature conjecture ( $R$ is always above some minimal value $R_{0}>0$ ) fits exceptionally well the $\Lambda$ CDM cosmological model [23]. These results were found by studying the quasi-Friedmannian equation, i.e. the dynamics of $L=f(R)$ theory; if one applies the dynamically equivalent Einstein frame it is easy to show that for any scale factor $a(t)$ there exists at least one function $f(R)$ having this factor as a solution of the field equation 4 [24]. Therefore there is no surprise that also by inclusion of corrections in a form of inverse squares of the Ricci and Riemann tensors the Supernovae data can be fitted without the need of any dark energy [26, 4]. On the other hand in the metric-affine formalism of gravity theories it was found that the current cosmological data (the supernovae Ia "gold set", the CMBR shift parameter and the linear growth factor) impose stringent restrictions on the test Lagrangian $L=R+\alpha R^{\beta}$ since $\beta \approx 0,05$ [27]; this result, however, does not uniquely confirm the $\Lambda$ CDM model and is rather due to the specific choice of the Lagrangian.

The main problem of cosmology which arises in this field of investigations is therefore

\footnotetext{
${ }^{4}$ Yet in a recent paper it is found that for a large class of the Lagrangians the evolution of linear density fluctuations does not fit the structure formation on both large and small scales simultaneously 25 .
} 
whether it is possible at all to effectively uniquely recover the underlying Lagrangian $L=f(R)$ from an exact analytic solution $a(t)$ to the quasi-Friedmannian equation. (Reconstruction of a Lagrangian depending on more than one curvature invariant is impossible.) The problem was investigated in more detailed way in two papers. First Capozziello et al. in an ingenious work [28] attempted to determine $f(R)$ assuming that one knows from observations a precise analytic dependence of the Hubble parameter $H$ on the redshift parameter $z, H(z)$. They expressed the curvature scalar $R$ as a function of $H(z)$ and then derived an equation for $f[R(z)]$ from the quasi-Friedmannian equation 5 . The equation for $f$ is a linear third order ODE with coefficients being extremely complicated functions of $H(z)$ and hence, in spite of its linearity, is analytically intractable. The authors were able to find out the initial data from observations for the equation so that there is a unique solution, but it is inaccessible. The method is unique and ineffective. It requires an exact analytic form of $H(z)$ while astronomic observations provide only a finite set of values $H(z)$ which are affected by large errors. To circumvent this difficulty the authors used a couple of simple functions approximating the real dependence of $H$ on $z$ which arise in recent models for dark energy such as quintessence and the Chaplygin gas. Given these analytic expressions, the numerical integration of the equation for $f[R(z)]$ is inevitable, then the solution is approximated by an empirical function. The final analytic fit for $f(R)$ looks rather unconvincing (eq. (48) of [28]) and the authors caution the reader against drawing any physical implications from it. Recently Fay et al. [29] have attempted to search for the form of $f(R)$ corresponding to the $\Lambda \mathrm{CDM}$ cosmology applying the general autonomous system for the $k=0 \mathrm{R}-\mathrm{W}$ spacetime. They found, among other functions, $L=R-2 \Lambda$, but this Lagrangian corresponds to saddle points of the autonomous system while the attractors of the system do not reproduce the radiation and the matter eras of the universe and give rise to a complicated irrational function $f(R)$. Their work clearly shows that the spatially flat $\mathrm{R}-\mathrm{W}$ spacetime is particularly deceptive in this respect. In fact, the dynamics of this spacetime is not so simple as is usually assumed. We almost never employ the exact solutions of the Friedmann equation for the real matter content of the universe as they are too involved and we are so accustomed to the approximate solutions (which very well fit the observations) that we view them as exact ones. For example, the period when the energy densities of the "radiation" (all the ultrarelativistic particles including neutrinos) and of the "dust matter" (nonrelativistic baryons and possibly the dark matter) were comparable was longer than the whole radiation era and in this period $a(t)$ was very complicated, nevertheless it is commonly assumed that in the radiation era there was no dust and this era was suddenly transformed into the matter era and after the transition the radiation disappeared. Under this assumption one gets the simple expressions $t^{1 / 2}$

\footnotetext{
${ }^{5}$ The evolution equation for $a(t)$ may be transformed into an equation for $a(z)$ and then for $f[R(z)]$ provided $z(t)$ is a monotonic function. As discussed above this means that one takes into account only this class of solutions. It is unclear as to what extent this restriction affects the final outcome.
} 
and $t^{2 / 3}$ for $a(t)$ in these eras respectively; this is the basic assumption in [29].

It should be stressed that in general it is impossible to reconstruct a differential equation from its approximate solution. A given function is an approximate solution to a whole class of equations and the correct equation is in no way distinguished within this class: the Einstein equations correspond to the saddle points rather than to attractors of the dynamical system of ref. [29]. Metric NLG theories are so conceptually involved that this (rather obvious) fact goes overlooked. As an example we take a simpler theory. Recall that it is possible to reconstruct the Newtonian theory (Laplace equation) from the exact solution, the Newton's potential. Now one tries to reconstruct it from an approximate solution. Suppose an astronomer observes a planet of mass $m$ moving on a very elongated elliptic orbit with the eccentricity $e=1-\varepsilon, \varepsilon \ll 1$ and $\varepsilon$ positive. He assumes that the gravitational force comes from a central potential $U(r)$ and tries to determine it from the planet's motion. To this end he employs the energy integral

$$
E=\frac{m \dot{r}^{2}}{2}+\frac{J^{2}}{2 m r^{2}}+U(r)
$$

where $J$ is the conserved angular momentum. The astronomer determines from observations the positions $r$ at different times and fits the data by an analytic function $r(t)$, inverts it and expresses the derivative $\dot{r}$ as a function $F(r)$. He makes his observations in two regimes: when the planet is close to perihelion and to aphelion. From the exact solution of the Kepler problem we may compute that near the perihelion the dependence $t(r)$ for this elongated orbit is approximately (by keeping only the dominant term in $\varepsilon$ )

$$
t \approx \frac{1}{3} \sqrt{\frac{2 m}{\alpha}} r^{3 / 2}
$$

for $U=-\frac{\alpha}{r}$ and this function should be used by the astronomer as an analytic fit to his data set. When the planet moves near the aphelion the approximate solution $t(r)$ which also fits the other observational data is

$$
t \approx \sqrt{\frac{m a^{3}}{\alpha}}\left(\pi-4 \sqrt{1-\frac{r}{2 a}}\right) .
$$

Both the functions are easily invertible and each is used to compute $\dot{r}=F(r)$. The quantities $a$ (the semimajor axis), $e, J$ and the parameter $p$ are determined from the observations (assuming ellipticity of the orbit), then $\alpha=\frac{J^{2}}{m p}$ and upon inserting $F(r)$ into the energy integral the astronomer finally finds that for $r$ close to $r$ min

$$
U(r) \approx-\frac{J^{2}}{m p r}-\frac{J^{2}}{2 m r^{2}}+E
$$

\footnotetext{
${ }^{6}$ In conformity with classical mechnics we denote it by $U$ instead of $\phi$.
} 
while close to the aphelion

$$
U(r) \approx-\frac{J^{2}}{4 m p a^{2}}(2 a-r)-\frac{J^{2}}{2 m r^{2}}+E .
$$

None of these expressions is an approximation to the Newton's potential. An ultimate conclusion from this example and the two works mentioned above is that reconstructing the underlying Lagrangian from known Robertson-Walker solutions is impossible for fundamental reasons. Relativistic metric theories of gravity are too intricate. It is in order here to remind the Einstein's view that a new physical theory is never formulated by induction from a pile of empirical data.

All that above does not imply that alternative theories of gravity should not be applied in cosmology and in particular should not be used to account for the dark energy. Today the situation in gravitational physics is exceptional as compared to other branches of physics: the well established and confirmed theory, general relativity, seems to be just a point in the "space" of all existing and conceivable theories of gravitational interactions and its nearest neighbourhood is densely populated by its alternatives, the metric NLG theories. According to Cantor's theorem the cardinal number of the set of metric NLG theories is greater than the cardinality of the continuum. The very existence of these theories entitles one to apply them to describe effects which are gravitational or may be interpreted as such. On the other hand the wealth of these theories makes necessary, before making any applications of one chosen from this huge set, to investigate two problems: i) to determine all possible interrelations between them and their relationships to general relativity, ii) whether a given gravity theory satisfies all the well grounded general rules of classical field theory and has acceptable properties. These include:

- determination of particle contents (spectrum),

- existence of a stable maximally symmetric ground state,

- form of interactions with ordinary matter, i.e. which quantities are measurable.

A natural and obvious postulate is to require that a given gravity theory should have a Newtonian limit. It however creates conceptual problems. It is not sufficient to formally get in the linear approximation the Poisson equation for a scalar potential [30] $\Delta U=4 \pi G \rho+\Lambda$ or another equation appearing in the Newtonian gravity (such as the stellar hydrostatic equilibrium one [31]). Newtonian gravity is well defined as a small static perturbation of Minkowski spacetime and the postulate may be unambiguously formulated for those Lagrangians which admit the flat spacetime as a solution. In this class of theories the postulate may work as an effective discriminating condition. Yet the NLG theories are most interesting and attractive for cosmologists if their ground state solution is curved being de Sitter or anti-de Sitter space while the flat spacetime is excluded. In this case the very concept of Newtonian gravity in these spacetimes is controversial and there are conflicting results on the weak-field limit for different La- 
grangians. As far as we know the problem of the Newtonian limit is not convincingly solved even in general relativity in the presence of the cosmological constant (a precise definition of the limit is lacking). Following cosmologists we deal mainly with the theories which do not admit Minkowski space as a solution and at the present level of knowledge the Newtonian limit criterion cannot be applied to them.

Investigations of the second problem, i.e., to what extent the metric NLG theories are viable from the viewpoint of classical field theory are the main subject of this paper and the subsequent Paper II. In section 2 we count the number of degrees of freedom and briefly discuss the particle spectrum of a general NLG theory. The latter subject requires replacing the fourth-order Lagrange equations by dynamically equivalent second order ones for the resulting gravitational triplet of fields; this aim is achieved by the powerful method of Legendre transformations. This decomposition is crucial for all investigations of the theory. The triplet may be described in infinitely many different frames and the two most important ones and the problem of coupling ordinary matter to the gravity fields are presented in section 3. The controversial problem of which frame is physical does not affect the criteria of viability of various gravity theories. The most fundamental criterion is the existence of a stable maximally symmetric ground state solution and it is investigated in Paper II. Conclusions and further considerations concerning the possibility of recovering the underlying $L=f(R)$ Lagrangian from a given (cosmological) solution are contained in section 4.

\section{Particle spectrum}

We consider in this section the general metric NLG theory based on an arbitrary Lagrangian $L=f\left(g_{\mu \nu}, R_{\alpha \beta \mu \nu}\right)$. The theory is metric in the sense that a nondegenerate tensor field $g_{\mu \nu}$ with Lorentzian signature is the only independent dynamical quantity. One may also investigate a metric-affine theory ("the Palatini method") with the same Lagrangian wherein one takes independent variations of $L$ with respect to the metric and a symmetric connection. As is well known, for any $L$ different from $R$ the two theories diverge. Some authors claim that the metric-affine approach is more natural since the Lagrange equations are of second order while in the purely metric theory they are of fourth order. However the metric NLG theory is not inherently a higher derivative one. The tensor $g_{\mu \nu}$ appearing in the Lagrangian actually is a kind of unifying field mixing various particles (fields) with different spins and masses. To find out a physical interpretation of the field it is necessary to decompose it in a multiplet of these fields. Then equations of motion for the separate fields are of second order and display a physical content of the theory better than the original fourth-order ones. This is why referring to NLG theories as "higher derivative ones" is misleading. In this respect the metric-affine approach is not advantageous over the purely metric formalism and we prefer the latter 
as conceptually simpler.

To avoid any confusion and for sake of completeness we begin with determining the degrees of freedom of the general metric NLG theory though it may be found in the literature. Counting the degrees of freedom (d.o.f.) in this case is far from trivial. The quadratic theory, $L=R+R^{2}+R_{\mu \nu} R^{\mu \nu}$, is known to have eight d.o.f. [1, 32, 33, 34]. For Lagrangians with arbitrary dependence on the Ricci tensor and applying a perturbative approach to Lagrangians depending on the Weyl tensor the d.o.f. were first counted by Hindawi et al. 35. by using a second order version of the theory. For most general Lagrangians it is possible to determine the maximal number of d.o.f. in the initial fourth-order formulation. It is well known that the pure gravitational field in general relativity, $R_{\mu \nu}=0$, has two d.o.f. [36] and we shall count them in the same way for $L=f\left(g_{\mu \nu}, R_{\alpha \beta \mu \nu}\right)$. The number of d.o.f. for a given system is defined as a half of the number of arbitrary functions needed to uniquely specify the initial data for the Cauchy problem for the equations of motion of the system. The equations of motion following from this Lagrangian form a system of ten tensor PDE of fourth order $E^{\alpha \beta}=0$ for the unifying field $g_{\mu \nu}$. Let a spacelike hypersurface $\mathrm{S}$ be chosen as an initial data surface for the equations. The theory is generally covariant (diffeomorphism invariant) and one can freely choose a coordinate system in the spacetime and the most convenient one is the comoving system (normal Gauss coordinates), $g_{00}=-1, g_{0 i}=0$, such that $\mathrm{S}$ has an equation $t=0$. There are six unknown functions $g_{i k}$ and the initial Cauchy data consist of values of $g_{i k}, \partial g_{i k} / \partial t, \partial^{2} g_{i k} / \partial t^{2}$ and $\partial^{3} g_{i k} / \partial t^{3}$ on $\mathrm{S}$; these are 24 functions of three coordinates $x^{i}$. The data are subject to a number of constraints. First, the coordinates on $\mathrm{S}$ may be freely changed and this gives a freedom of choice of 3 functions of these variables. Secondly, the trace of the extrinsic curvature of $S$ may be given any value, i.e. one function is arbitrary. Finally, the general covariance of the theory implies, in the same way as in general relativity, that some of the field equations are constraints. In fact, the invariance of the action

$$
S=\int \mathrm{d}^{4} x \sqrt{-g} f\left(g_{\mu \nu}, R_{\alpha \beta \mu \nu}\right)
$$

under an infinitesimal coordinate transformation gives rise to a strong Noether conservation law ("a generalized Bianchi identity") $\nabla_{\beta} E_{\alpha}^{\beta} \equiv 0$ (see e.g. appendix A in arXiv version of [37]). Explicitly it reads

$$
\partial_{0} E_{\alpha}^{0}+\partial_{i} E_{\alpha}^{i}+\Gamma_{\beta \nu}^{\beta} E_{\alpha}^{\nu}-\Gamma_{\beta \alpha}^{\nu} E_{\nu}^{\beta}=0 .
$$

The last three terms contain at most fourth time derivatives of $g_{\mu \nu}$ and the identity implies that $\partial_{0} E_{\alpha}^{0}$ cannot involve fifth time derivatives. Thus $E_{\alpha}^{0}$ involve at most third time derivatives and $E_{\alpha}^{0}=0$ are not propagation equations but form four constraints on the initial data. Together the number of independent Cauchy data is diminished to 16 
arbitrary functions and thus a general metric NLG theory has eight d.o.f.

For a restricted NLG theory, $L=f(R)$, the number of d.o.f. is less than 8 what means that the equations $E_{\beta}^{\alpha}=0$ generate additional constraints on the Cauchy data. It is not easy to determine all the constraints from these equations and one should instead apply a second order formulation of the theory. To this end it is adequate to view the $L=f(R)$ theories in a wider context of as large class of of NLG theories as possible. We therefore consider for the time being Lagrangians $L=f\left(g_{\mu \nu}, R_{\alpha \beta}\right)$, i.e. with no dependence on the Weyl tensor.

An adequate mathematical tool for this purpose is provided by a specific Legendre map [38, 39, 40]. For these NLG theories the method is as general and powerful as Legendre maps transforming the Lagrangian formalism into the Hamiltonian formalism in classical mechanics and classical field theory. Yet the method is not currently used in a systematic way and most papers on applications in cosmology have employed various ad hoc tricks to transform from the Jordan frame to the Einstein frame. The tricks in most cases give results equivalent to the Legendre transformation, however do not allow to fully display the structure and features of the theory. Here we give a brief summary of investigations of particle spectrum contained in [41] while the general formalism is described in [38, 39, 40].

The Jordan frame consists of only one dynamical variable, the tensor field $g_{\mu \nu}, \mathrm{JF}=$ $\left\{g_{\mu \nu}\right\}$, which plays both the role of a metric tensor on a spacetime $M$ and a kind of unifying gravitational field being a composition of some fields having definite spins and masses. Pure gravity is then described by a multiplet of the fields having together at most eight d.o.f., the metric is a geometric component of the multiplet. The unifying field may be decomposed into the component physical fields in two ways. The first method assumes that $g_{\mu \nu}$ is the spacetime metric and one separates from it, by means of a Legendre map, the additional degrees of freedom, i.e. the other components of the multiplet. The Ricci tensor is decomposed into its irreducible parts, the trace $R$ and the traceless tensor $S_{\mu \nu} \equiv R_{\mu \nu}-\frac{1}{4} g_{\mu \nu} R$, then one defines a scalar and a tensor canonical momentum conjugate to the "velocity" $R_{\mu \nu}$ by Legendre transformations

$$
\chi+1 \equiv \frac{\partial L}{\partial R}, \quad \pi^{\mu \nu} \equiv \frac{\partial L}{\partial S_{\mu \nu}}=\pi^{\nu \mu} .
$$

Together with the metric the two fields form a gravitational triplet, named the HelmholtzJordan frame, HJF $=\left\{g_{\mu \nu}, \chi, \pi^{\mu \nu}\right\}$. Equations of motion for the triplet follow from a Helmholtz Lagrangian $L_{H}$ which is dynamically equivalent to the initial $L=f\left(g_{\mu \nu}, R_{\alpha \beta}\right)$. Here one meets a technical obstacle: to get an explicit form of $L_{H}$ one must express $R$ and $S_{\mu \nu}$ in terms of $\chi$ and $\pi^{\mu \nu}$, i.e. to solve the defining equations to find $R=r\left(\chi, \pi^{\mu \nu}\right)$ and $S_{\mu \nu}=s_{\mu \nu}\left(\chi, \pi^{\alpha \beta}\right)$. For a general $L=f\left(g_{\mu \nu}, R_{\alpha \beta}\right)$ this requires solving nonlinear matrix equations. (No doubt, the power of Hamiltonian formalism in physics stems 
from the fact that physically relevant Lagrangians are quadratic in "velocities".) This is why only Lagrangians that are quadratic in the Ricci tensor have been investigated in detail. However the formalism in principle works for any $L$. Furthermore, as we shall see, in the Einstein frame the specific dependence of $L$ on $R_{\mu \nu}$ only affects interaction terms while the general structure of the theory remains unaffected, therefore although a general formalism (in HJF) has been developed for any function $f$, in practice one applies only those $f$ for which the Legendre transformations may be effectively inverted. For any $f$ the Helmholtz Lagrangian reads

$$
L_{H}=R+\chi R+\pi^{\mu \nu} S_{\mu \nu}-H\left(\chi, \pi^{\mu \nu}\right),
$$

where $H$ is a Hamiltonian. This Lagrangian is linear in $R_{\mu \nu}$, what implies that Lagrange equations for $g_{\mu \nu}$ take form of Einstein field equations, $G_{\mu \nu}=8 \pi G T_{\mu \nu}\left(R_{\alpha \beta}, \chi, \pi^{\alpha \beta}\right)$. The RHS of these equations is by definition an energy-momentum tensor for the two fields and it depends linearly on the Ricci tensor and on the first and second derivatives of $\chi$ and $\pi^{\mu \nu}$. There are no kinetic terms for $\chi$ and $\pi^{\mu \nu}$ in $L_{H}$ and propagation equations for the fields are derived in a rather intricate way from $T_{\mu \nu}$, these are hyperbolic second order ones. Both the fields are subject to one algebraic and four first order differential constraints and in consequence they carry together six degrees of freedom. In summary, the particle spectrum of the theory exhibited in HJF consists of: a massless spin-2 field (graviton7, spin two and 2 d.o.f.), a massive spin-2 field (5 d.o.f.) and a massive scalar field. This outcome (with the same values of the masses for the two fields) was first found in the linear approximation [1, 43] and then by various methods in the exact theory for a quadratic Lagrangian $R+R^{2}+R_{\mu \nu} R^{\mu \nu}$ [44, 45]. HJF is not uniquely determined: since the Helmholtz Lagrangian in (1) is not in a canonical form, various redefinitions of $\chi$ and $\pi^{\mu \nu}$ are admissible.

Introducing the field $\pi^{\mu \nu}$ makes sense if its definition may be (at least in principle) inverted to yield $S_{\mu \nu}=s_{\mu \nu}\left(\chi, \pi^{\alpha \beta}\right)$, otherwise $\pi^{\mu \nu} \equiv 0$. The field exists if the Lagrangian depends on $R_{\mu \nu}$ in a nontrivial way, i.e. the Hessian

$$
\operatorname{det}\left(\frac{\partial^{2} f}{\partial R_{\alpha \beta} \partial R_{\mu \nu}}\right) \neq 0 .
$$

This condition does not hold for $L=f(R)$. Then $\pi^{\mu \nu}$ vanishes and gravity is described by a doublet $\mathrm{HJF}=\left\{g_{\mu \nu}, \chi\right\}$ carrying $2+1$ d.o.f. and the scalar cannot be massless. Thus for this class of Lagrangians determining the particle spectrum is very simple and

\footnotetext{
${ }^{7}$ We use this traditional name for the metric field satisfying $R_{\mu \nu}=0$. It is worth noting, however, that the relationship between the hypothetical quantum of the gravitational field and the classical field (described by general relativity) is different from that between the photon and the classical Maxwell field, see [42].
} 
straightforward.

The other approach to constructing a second order formalism is more sophisticated. Here one assumes that $g_{\mu \nu}$ is merely a unifying field for gravitation and plays a role of a spacetime metric in a purely formal way - in the sense that the Ricci tensor appearing in the Lagrangian is made up of it. One introduces a new metric as a canonical momentum conjugate to the full Ricci tensor via a Legendre map as [38, 39]

$$
\tilde{g}^{\mu \nu} \equiv(-g)^{-1 / 2}\left|\operatorname{det}\left(\frac{\partial f}{\partial R_{\alpha \beta}}\right)\right|^{-1 / 2} \frac{\partial f}{\partial R_{\mu \nu}},
$$

here $g=\operatorname{det}\left(g_{\mu \nu}\right) . \tilde{g}^{\mu \nu}$ may be viewed as a metric tensor providing that $\operatorname{det}\left(\partial f / \partial R_{\alpha \beta}\right) \neq$ 0 . Clearly for $f(R)=R$ one gets $\tilde{g}^{\mu \nu}=g^{\mu \nu}$ and for arbitrary $f(R)$ the new metric is conformally related to the old one, $\tilde{g}_{\mu \nu}=f^{\prime}(R) g_{\mu \nu}$, where $\tilde{g}_{\mu \nu}$ is the matrix inverse to $\tilde{g}^{\mu \nu}$. In this case the Legendre transformation is degenerate since it cannot be inverted. In general the transformation is a map of a (Lorentzian) metric manifold $\left(M, g_{\mu \nu}\right)$ into another one, $\left(M, \tilde{g}_{\mu \nu}\right)$. If the transformation (2) is invertible, i.e. the Hessian for $f$ does not vanish, it may be solved to give $R_{\mu \nu}=r_{\mu \nu}\left(g_{\alpha \beta}, \tilde{g}^{\lambda \sigma}\right)$. As in Helmholtz-Jordan frame one constructs a Helmholtz Lagrangian which now takes a generic form

$$
\tilde{L}_{H}=\tilde{R}(\tilde{g})+K(\tilde{\nabla} g)-\tilde{g}^{\mu \nu} r_{\mu \nu}(g, \tilde{g})+\left|\frac{\operatorname{det}\left(g_{\alpha \beta}\right)}{\operatorname{det}\left(\tilde{g}_{\alpha \beta}\right)}\right|^{1 / 2} f\left(g_{\mu \nu}, r_{\alpha \beta}(g, \tilde{g})\right),
$$

where $\tilde{\nabla}$ is the covariant derivative with respect to $\tilde{g}_{\mu \nu}$. It is worth stressing that in Einstein frame, $\mathrm{EF}=\left\{\tilde{g}_{\mu \nu}, g_{\alpha \beta}\right\}$, precisely the Einstein-Hilbert Lagrangian for the spacetime metric $\tilde{g}_{\mu \nu}$ is recovered, giving rise to Einstein field equations $\tilde{G}_{\mu \nu}(\tilde{g})=$ $8 \pi G \tilde{T}_{\mu \nu}(\tilde{g}, g)$ with the tensor field $g_{\mu \nu}$ acting as a "matter" source for the metric. The tensor $\tilde{T}_{\mu \nu}$ is the variational energy-momentum tensor for $g_{\mu \nu}$ defined in the standard way and contains second derivatives $\tilde{\nabla}_{\mu} \tilde{\nabla}_{\nu} g_{\alpha \beta}$ but no curvature 8 . Unlike $L_{H}$ in HJF, the Lagrangian has a canonical form for $g_{\alpha \beta}$, i.e. is a sum of a kinetic term and a potential part. The kinetic term, $K(\tilde{\nabla} g)$, is a quadratic polynomial in first derivatives $\tilde{\nabla}_{\mu} g_{\alpha \beta}$, as is usual in classical field theory and what is really remarkable, it is universal, i.e. is independent of the form of the function $f[38,39$. The only reminiscence of the original $L$ in JF is contained in the potential part of $\tilde{L}_{H}$ : explicitly via $f\left(g_{\mu \nu}, r_{\alpha \beta}\right)$ and implicitly via $r_{\alpha \beta}$, i.e. in interaction terms. Hence in $\mathrm{EF}$ one recovers just general relativity with

\footnotetext{
${ }^{8}$ In general the energy-momentum tensor involves second derivatives of matter variables, the gauge fields, the minimally coupled scalar field and perfect fluids belong to few exceptions.

${ }^{9}$ Clearly this is not little. In quantum mechanics every state vector satisfies the Schrödinger equation and the whole variety of quantum systems is encompassed in interaction terms in the Hamiltonian. Here something analogous occurs. We stress this point since in the fourth-order formulation of an NLG theory in JF an impression arises that the theory is more different from general relativity than it indeed is.
} 
a source which may be interpreted either as a nongeometric component of gravity or merely as a (quite exotic) matter field described by classical field theory. In this sense Einstein general relativity is a universal Hamiltonian image (under the Legendre map) of any $L=f\left(g_{\mu \nu}, R_{\alpha \beta}\right)$ gravity theory. In other terms, general relativity is an isolated point in the space of all gravity theories: its closest neighbourhood, consisting of the metric NLG theories, can be mapped onto it and thus is not different from GR. It is also clear that in practice there is no need in studying Lagrangians more complicated than quadratic in $R$ and $R_{\mu \nu}$.

The second order Lagrange equations for $g_{\mu \nu}$ in EF are subject to four differential constraints (following from Bianchi identities $\nabla_{\nu} G_{\mu}^{\nu}(g)=0$ ) which allow one to eliminate four of ten components of the field. This shows that it carries six d.o.f. and is actually a mixture of two different physical fields. The next step is thus to decompose it into components with definite spins. Then one again gets a scalar and a spin-2 field. For practical purposes it is convenient to eliminate the scalar from the outset by an appropriate choice of the Lagrangian in JF [41]. The field $\psi_{\mu \nu}$ arising in this way from $g_{\mu \nu}$ carries spin two (and five d.o.f.), has the same mass as that computed in HJF for $\pi^{\mu \nu}$ and is nonlinear (it is well known that any linear spin-2 field is inconsistent in general relativity [46]). It is straightforward to show in EF that $\psi_{\mu \nu}$ is necessarily a ghost field (a "poltergeist") [1, 43, 44, 45, 47], while it is rather difficult to establish this feature for $\pi^{\mu \nu}$ in HJF. And to avoid any misunderstanding we stress that the ghost-like behaviour of the spin-2 field is inevitable: it appears in any consistent theory of gravitationally interacting spin-2 fields [48] and in particular is a feature of any $L=f\left(g_{\mu \nu}, R_{\alpha \beta}\right)$ gravity.

All these gravity theories are similar and they differ only in the interaction terms in (3) and in masses of the spin-2 and scalar components of the gravitational triplet. All the $L=f(R)$ theories are reduced in Einstein frame to general relativity plus a massive minimally coupled field with a self-interaction potential determined by $f$ [37]. At first sight it seems that Lagrangians generating tachyonic masses of the fields should be excluded as untenable. This is the case when the ground state solution is Minkowski space and other fields behave as small excitations in this spacetime. However, if the ground state is anti-de Sitter, also the scalar field with a tachyon mass is allowed if its modulus is not too large in comparison to the cosmological constant, see Paper II.

The case of most general Lagrangians, $L=f\left(g_{\mu \nu}, R_{\alpha \beta}, C_{\mu \nu \alpha \beta}\right)$, explicitly depending on the Weyl tensor, is more subtle. The unifying field $g_{\mu \nu}$ carries 8 d.o.f. and one conjectures that it can be decomposed in the same triplet of the graviton, a spin-2 field and a scalar, however a proof for an arbitrary $L$ is missing. The conjecture was proved only in a perturbative analysis (and in four dimensions): one expands a generic Lagrangian about a ground state solution of the theory (Minkowski, de Sitter or anti-de 
Sitter) up to terms quadratic in Riemann tensor and arrives at [35, 49]

$$
L=\text { const }+R+a R^{2}+b R_{\mu \nu} R^{\mu \nu}+c \mathrm{~GB},
$$

where GB, the Gauss-Bonnet term, is a topological invariant and in $d=4$ may be discarded as a divergence. Thus the most general NLG theory perturbatively reduces to that without the Weyl tensor and has the same particle spectrum.

We stress again that in both the frames any NLG theory is reduced to general relativity plus some exotic source. In EF the general-relativistic form of the theory is obvious, in HJF it is less conspicuous due to the specific form of the Helmholtz Lagrangian (1). From the physical viewpoint the spin-two and spin-0 fields may be viewed either as the components of the gravitational triplet or just as a kind of matter. It is not quite clear whether the difference between the two interpretations is empirical: whether there is a "gedankenexperiment" allowing to differentiate one from the other. As in Brans-Dicke theory it is necessary to assume that the two fields do not couple to any other matter in the sense that in a relevant Lagrangian there are no interaction terms of the two fields with the particles of the standard model. And as in Brans-Dicke theory "one way" interactions are admissible: ordinary matter may act as a source in equations of motion for $\chi$ and $\pi^{\mu \nu}$. The two nongeometric components of gravity only interact gravitationally in the sense that there is interaction between them and, first of all, they act as a source of the spacetime metric in Einstein field equations. It is often assumed (and clearly there is no proof) that the dark energy signals its existence solely by its influence on the cosmic evolution. Whether it should be regarded as a form of matter or as a component of gravity is presently a matter of convention. Mathematically the issue is irrelevant and Einstein field equations arising from (1) and (3) should be studied by applying all the methods developed to this aim in general relativity. In particular, the energy-momentum tensors for the spin- 0 and spin-2 fields appearing in these equations ought to satisfy the conditions usually imposed on matter in general relativity. Clearly it may be claimed that the two components of gravity are specific in the sense that they need not satisfy the standard conditions, e.g. the scalar field may not be subject to energy conditions. It might be so, however the price would then be high: the most significant results (or their appropriate analogues) found in general relativity would be inaccessible in a given NLG theory. In this work we assume that the spin-0 gravity is not exceptional in this respect.

\section{Frames and initial conditions}

Once an NLG theory is expressed in HJF one may perform arbitrary Legendre transformations ("canonical ones") and changes of variables (field redefinitions), thus the theory may be formulated in infinitely many various frames. Clearly both HJF and 
EF are priviledged by their construction exhibiting the physical content of the theory. All the frames are mathematically equivalent provided the transformations are at least locally invertible (proving a global invertibility is a hard task) and nonsingular. Equivalence means that the space of solutions in one frame is in a one-to-one correspondence to the space of solutions in another frame. The corresponding solutions are different and physical quantities made up of them are different, most notably energy is very sensitive to various transformations. Thus dynamical equivalence of frames implies their physical inequivalence. As long as the theory is closed, i.e. all that exists is contained in a Lagrangian of the theory (in the case discussed here "everything that exists" is pure gravity and there is no matter), this inequivalence is irrelevant as undetectable. All the frames are equally physical. For example, spacetime intervals between a given pair of events are different in distinct frames and the differences cannot be measured without external rods and clocks. And to measure energy of the gravitational triplet one needs an external device which is not included in the Lagrangian, yet in a closed theory neither an external observer nor external device does exist. In the same way spacetime intervals between a given pair of events are different in different frames and their differences cannot be measured without external rods and clocks. In this sense all the theories of physics are open: the observer and his equipment is not described by a tested theory 10. To make an NLG theory open it is necessary to couple it to ordinary matter and predict then some effects which may be observed by an external agent.

Coupling of matter to gravity should proceed in the same way as in general relativity where, however, no ambiguity appears since there is only one frame. For concreteness we consider now the restricted NLG theories, $L=f(R)$, since we will be dealing with them in Paper II. Clearly the coupling of matter to gravity is the same in any NLG theory. One takes pure gravity and chooses a frame consisting of a tensor $\gamma_{\mu \nu}$ regarded as a spacetime metric and a scalar $\phi$; these quantities are some functions of the variables $g_{\mu \nu}$ and $\chi$ forming HJF. The corresponding Lagrangian may have almost arbitrary form $L_{g}(R(\gamma), \phi)$ [44, 37]. For a given kind of matter $\Psi$ its Lagrangian is constructed in special relativity and gets some form $L_{m}\left(\eta_{\mu \nu}, \Psi, \partial \Psi\right)$. By definition, $\gamma_{\mu \nu}$ is the metric of the physical spacetime and any matter minimally couples to it, hence the matter Lagrangian for $\Psi$ becomes $L_{m}\left(\gamma_{\mu \nu}, \Psi, \nabla \Psi\right)$, where $\nabla$ is the Levi-Civita connection for $\gamma_{\mu \nu}$. The scalar gravity $\phi$ does not couple to the matter and the total Lagrangian is just $L_{t}=L_{g}+L_{m}$. The chosen frame is the physical frame due to the minimal coupling. In all other frames where the transformed tensor playing the role of a metric is different from $\gamma_{\mu \nu}$, the matter is nonminimally coupled to it and a coupling to $\phi$ may appear. Dynamical equivalence of various frames remains preserved in presence of any matter while these frames should be regarded as unphysical since experimental devices

\footnotetext{
${ }^{10}$ This is not so trivial as it may seem. There are some tendencies in quantum gravity to regard it as a closed theory.
} 
measure quantities made up of variables of the physical frame. For example, optical observations disclose that the light of distant galaxies is redshifted, what is interpreted as that expanding Robertson-Walker spacetime forms the physical frame, while one may make all computations in a conformally related frame where the spacetime is flat. In this case outcomes of the computations must be transformed back to the physical frame, the $\mathrm{R}-\mathrm{W}$ spacetime, if they are to be confronted with observations. There is nothing new in this, the same is always done in classical mechanics: first one determines (experimentally) physical positions and momenta of a given system, makes a canonical transformation mixing these quantities to new variables in which the Hamilton equations are easiest solvable and finally makes the inverse transformation to express a given solution in physical variables. In classical mechanics this is obvious, in a gravity theory it is not.

Assume, as most authors applying NLG theories to cosmology actually do, that JF is physical, then the total Lagrangian is $L_{t}=f(R)+L_{m}\left(g_{\mu \nu}, \Psi, \nabla \Psi\right)$ with $\nabla$ being now the metric connection for $g_{\mu \nu}$. The Helmholtz Lagrangian in HJF reads

$$
L_{H}=p[R(g)-r(p)]+f(r(p))+L_{m}\left(g_{\mu \nu}, \Psi, \nabla \Psi\right),
$$

from now on the spin- 0 gravity in HJF is denoted $p$ and defined as $p \equiv \frac{\mathrm{d} f}{\mathrm{~d} R}$ in conformity with [38, 37]. The Lagrange equations are then $R(g)=r(p)$,

$$
\begin{gathered}
G_{\mu \nu}(g)=\theta_{\mu \nu}(g, p)+\frac{1}{p} t_{\mu \nu}(g, \Psi) \\
\text { and } \quad \frac{\delta L_{m}}{\delta \Psi}=0 .
\end{gathered}
$$

Here $r(p)$ is a (possibly unique) solution of the equation $\frac{\mathrm{d} f(r)}{\mathrm{d} r}=p$ and $\theta_{\mu \nu}$ is an effective energy-momentum tensor for $p$ [37] while $t_{\mu \nu}(g, \Psi)$ is the standard variational energymomentum tensor for matter derived from $L_{m}$ with the aid of $g_{\mu \nu}$. A propagation equation for the scalar is derived, as previously for $\chi$, from the tensor $\theta_{\mu \nu}$ by taking the trace of (5) and it reads

$$
\square p-\frac{2}{3} f(r(p))+\frac{1}{3} p r(p)=-\frac{1}{3} g^{\mu \nu} t_{\mu \nu}(g, \Psi) .
$$

It is easy to see that the scalar does not have its own Lagrangian and eq. (7) must be derived in this roundabout way. The metric field has two sources, the matter and spin-0 gravity. The equation of motion for any matter, (6), is independent of $p$, yet the matter forms a source term $g^{\mu \nu} t_{\mu \nu}$ for the scalar gravity. In this sense the field $p$ does not directly affect motions of matter and its effects are confined to affecting the metric via eq. (5). 
The initial and/or boundary conditions for both $g_{\mu \nu}$ and $p$ are related to or even determined by a matter distribution. For example, in the solar system the matter distribution is dominated by the sun and both the fields are static spherically symmetric. Inside the sun the strength of the material source for $g_{\mu \nu}$ and $p$ is comparable and both the fields are there relatively strong and regular at the centre. Outside the sun the fields are fading towards spatial infinity. Notice that the scalar generates spherically symmetric corrections to Schwarzschild solution. Search for these corrections has resulted in the fact that the Lagrangian $R+1 / R$ is ruled out by measurements of the PPN parameter $\gamma$ in the solar system [12 and provides very stringent bounds on the size of possible corrections to the Einstein-Hilbert Lagrangian $L=R$ [19, 7, 16]. This is an indication that Jordan frame is unlikely to be the physical frame.

If instead, Einstein frame is regarded as physical, the Helmholtz Lagrangian takes a form well known from general relativity,

$$
\tilde{L}_{H}=\tilde{R}(\tilde{g})-\tilde{g}^{\mu \nu} \phi_{, \mu} \phi_{, \nu}-2 V(\phi)+L_{m}(\tilde{g}, \Psi, \tilde{\nabla} \Psi),
$$

where $\phi \equiv \sqrt{\frac{3}{2}} \ln p, V$ is a potential determined by $f$ and matter is minimally coupled to $\tilde{g}_{\mu \nu}$. The Lagrangians $L_{m}$ in (4) and (8) have the same dependence on the spacetime metric, $g_{\mu \nu}$ and $\tilde{g}_{\mu \nu}$, respectively. As a consequence the matter energy-momentum tensor in $\mathrm{EF}$ is just $t_{\mu \nu}(\tilde{g}, \Psi)$. The field equations are now directly derived as the variational ones,

$$
\begin{gathered}
\tilde{G}_{\mu \nu}(\tilde{g})=T_{\mu \nu}(\tilde{g}, \phi)+t_{\mu \nu}(\tilde{g}, \Psi) \\
\widetilde{\square} \phi=\frac{\mathrm{d} V}{\mathrm{~d} \phi} \\
\text { and } \quad \frac{\delta L_{m}}{\delta \Psi}=0 .
\end{gathered}
$$

Obviously one obtains eq. (11) from (6) by replacing $g_{\mu \nu}$ with $\tilde{g}_{\mu \nu}$. Now the scalar gravity is completely decoupled from matter and solely interacts with the metric field. This implies that initial and boundary conditions for $\phi$ are independent of matter distribution. The metric has two independent sources and its symmetries, boundary and initial conditions are determined by both or by the source that dominates. For example, in the solar system the $\phi$ field need not be spherical. From the spherical symmetry of the local spacetime one infers that the matter (the sun) is dominant on the RHS of eq. (9) and any small deviations from Schwarzschild metric should be nonspherical. The scalar field has no local (matter or any other) sources and fills the entire universe and approximately is described by one global solution. The solution is approximate since the scalar interacts and is affected by the spacetime metric and the latter is affected by local matter inhomogeneities. This global solution which is realized in our universe was determined by some initial and boundary conditions near the Big Bang. From the 
observations showing that the cosmic space is homogeneous and isotropic on large scales it is inferred that either the $\phi$ field is homogeneous (and time dependent) throughout the spacetime since the Big Bang or it is inhomogeneous and undetectably (even using cosmological data) weak. Clearly the first possibility is more attractive as it may a priori account for the dark energy. In any case a solution for the scalar has been chosen once for the universe and unlike the metric field it cannot be locally varied according to local (arbitrary) conditions. In other words, even in vicinity of a black hole, where the spacetime is extremely distinct from the Robertson-Walker one, the $\phi$ field only to some extent deviates from its overall cosmological solution. We emphasize that if some nonspherical deviations from the Schwarzschild metric are detected in the solar system (after subtracting all effects of planets, the Kuiper belt, the Oort cloud etc.), they may be accounted for by the scalar component of gravity in Einstein frame.

Both the frames are experimentally distinct. Which of them (if any) is physical? In other terms, which metric is minimally coupled to matter? The problem arises in any theory in which various frames appear. For example, in string theory in the low energy field-theory limit of string action one may use either the string (Jordan) frame in which the stringy matter is minimally coupled to the metric while the dilaton field is nonminimally coupled to it or transform to the conformally related Einstein frame where the dilaton is minimally coupled to the new metric and has the canonical kinetic term. The two frames are usually considered as completely equivalent for describing the physics of the massless modes of the string. This is particularly noticeable in the pre-Big Bang inflationary string cosmology. A superinflationary solution in string frame becomes an accelerated contraction in Einstein frame and vice versa. This drastic difference in behaviour of the cosmic scale factor in both the frames is irrelevant for string cosmology. In fact, the number of strings per unit of string volume is decreasing in time during the pre-Big Bang inflation in both frames and the temperature of the string gas grows in comparison to the temperature of the photon gas in both frames [50]. Whether $a(t)$ is expanding or contracting, the horizon/flatness problem of cosmology may be solved in each frame.

This frame independence for the physical effects of inflationary solutions in string cosmology, showing invariance of physics under local field redefinitions, has been found, however, only for a limited number of observables among those which can be constructed in this theory. Other observables are frame dependent. Even in the early universe spacetime intervals are measurable, at least in principle if not in practice, and these quantities depend on whether $a(t)$ grows or decreases. The very fact that we observe that both the optical spectra of distant galaxies and the cosmic microwave background radiation are redshifted, indicates that cosmology is not frame independent. The pre-Big Bang era of string cosmology is so far from us and exotic that very few effects may be observed today and these turn out to be frame invariant. Yet it is doubtful if string theory 
as such is frame (i.e. conformally) invariant 11 . This would mean that the theory is closed.

In metric NLG theories which are closer to experimental physics and observational astronomy, the fact that most observables are frame dependent 12 , i.e. only one frame is physical (is unique up to trivial field redefinitions), is hardly arguable (see however references in [37]). The ultimate decision of which frame is physical will be given by experiment, but it should not be expected very soon. Before making any application of these theories it is necessary to establish by some theoretical arguments or by mere assumption which frame is measurable. More than ten years ago we studied in detail the full network of relationships between restricted NLG theories, scalar-tensor gravity theories and general relativity and effects of introducing matter in various frames and on this basis we gave arguments in favour of Einstein frame [37]. After our work there was much discussion in the literature on the subject [52]. Since the discovery that a modified gravity might replace the dark energy most authors have preferred the Jordan frame as physical. As it is motivated in a paper, "if one wants to consider modifications of gravity like scalar-tensor theory or metric $f(R)$ gravity, the Jordan frame should be assumed to be the physical one" since if Einstein frame is physical "the resulting theory will be no different from general relativity". These authors seem to be unaware that also in Helmholtz--Jordan frame the field equations for the metric tensor are Einstein ones. The assumption that by choosing Jordan frame as physical (and minimally coupling all matter in this frame) one gets a theory which is essentially more different from general relativity than in the other case, is a mere illusion.

We do not wish to enter the debate again. In our opinion all relevant arguments in favour of JF and EF have already been expounded. We only point out that in most cases the Einstein frame is computationally advantageous (and this is explicitly or implicitly acknowledged by most authors who implicitly or explicitly assume Jordan frame as physical). For example, the Zeroth Law and the Second Law of black hole thermodynamics for a polynomial $f(R)$ have been proved only in Einstein frame [53]. On the other hand we keep open mind for the possibility that the real world might be not so simple as we expect. It might be so that the real physics is in Jordan frame while Einstein frame remains advantageous both in solving equations of motion and, what is most important, in proving general features of a given theory. The issue will be resolved by experiment.

The reader may find these comments on physical interpretation of various frames as

\footnotetext{
${ }^{11}$ In string theory, which is still far from making concrete physical predictions, it is difficult to establish what is measurable. Some authors claiming complete frame invariance of the theory seem to confuse the dynamical equivalence of various frames, which is indisputable, with physical equivalence.

${ }^{12}$ Nevertheless in Jordan and Einstein frames many physical quantities are the same, e.g. for black holes all the thermodynamical variables do not alter under a suitable Legendre map [51].
} 
unnecessary. We explain that we make these comments explicitly and in some length since for many researchers in cosmology these things are far from being clear. The level of confusion may be best seen from the introduction to the work [54] where the authors express their surprise that the following procedure: i) transform the equations of motion from Jordan frame (which is assumed to be physical) to Einstein frame, ii) solve the transformed equations and choose these solutions which seem to be physically significant and iii) transform back the chosen solutions to JF, does not provide physically meaningful results. They even quote a paper where it is claimed that "passing from one frame to the other can change the stability of the solution" - a statement which for a mathematically oriented reader clearly means that the transformation is singular. The authors of 54] summarize their doubts in the question "is it correct to obtain a solution in a frame and then interpret it in another frame?". To leave no space for any ambiguity we respond: yes it is, in the same sense as it is done in classical mechanics where we deal with canonical transformations.

We emphasize that for the purposes of the present work the issue of which frame is physical, is irrelevant. We claim that the order of investigations should be as follows. First one chooses (on some basis) one or a class of NLG theories. Second, one verifies if the chosen theory is viable from the viewpoint of classical field theory. Third, a physical frame is assumed. Then equations of motion are solved either in JF or in HJF, EF or some other frame, depending on computational facilities (usually EF is most convenient). Finally the solutions should be transformed back to the physical frame (if were found in another one) to construct physical observables which will be confronted with observations. We stress that the second step cannot be passed over otherwise there is danger that the work will be wasted. In fact, in some papers long and nontrivial computations have been performed in a framework of a theory which is definitely untenable. Viability criteria are independent of the choice of the physical frame. This frame independence does not mean that whether a given theory is physically viable or not can be established in any frame. On the contrary, all the methods developed up to now to investigate the viability do work in Einstein frame and usually (besides one case mentioned in Paper II) do not work in most other frames. In this sense Einstein frame is mathematically distinguished. Yet these methods neither prove that this frame is physical nor assume it. We shall use Einstein frame for checking viability various $L=f(R)$ theories. Therefore the first, basic assumption or criterion a theory should pass is the existence of Einstein frame: the Legendre transformation from JF to EF must be regular (in a neighbourhood of a candidate ground state solution). Once a theory meets the criteria and is regarded tenable one may choose the physical frame. We shall see that there are infinitely many viable theories and untenable ones are equally numerous. Obviously of all viable restricted NLG theories only one (if any) is correct, i.e. will be fully confirmed by experiment and observations. By the time this occurs one may view any viable theory as a candidate for describing gravitational interactions in 
the nature.

Out of all possible viability criteria for a classical field theory the most appropriate (and effectively applicable) one in the case of metric NLG theories is the existence of a stable maximally symmetric ground state. Paper II is devoted to studying and applying this criterion.

\section{Conclusions}

It is reasonable to conjecture that the accelerated expansion of the universe is not driven by an extremely exotic and unknown to physics kind of matter with negative pressure but is rather due to some modification of gravitational interactions. However in search for a modified gravity theory great caution is necessary. Gravitational physics is exceptional among all branches of physics in that there is a great variety of competing theories, all of which are some variations of Einstein's general relativity. Modifications may go in all possible directions while in most cases gravity theories applied to cosmology differ from general relativity only in one axiom: the form of the field equations. The assumption that a gravitational Lagrangian is an arbitrary function $L=f\left(g_{\mu \nu}, R_{\alpha \beta \mu \nu}\right)$ gives rise to infinity of theories and choosing the correct one is a hard task. It is almost invariably attempted to make this choice employing the cosmic scale factor $a(t)$ in the spatially flat Robertson-Walker spacetime. This approach is actually hopeless even in the framework of restricted metric gravity theories with $L=f(R)$. It has been shown 28. that if one knows the exact analytic form of $a(t)$ and a number of initial conditions, then the function $f(R)$ is a unique solution of a linear third order ODE. This method does not work in practice since the equation is intractably complicated. The fundamental cause that the method cannot work is that the real physical spacetime is not $\mathrm{R}-\mathrm{W}$ one. The cosmic matter distribution becomes homogeneous and isotropic only asymptotically at large scales. If one were ingenious enough to solve the differential equation for $f(R)$ employing that form of $a(t)$ which best fits all the astronomical data, the resulting Lagrangian would be rather different from the true one. The standard Friedmannian cosmology based on general relativity is the best fit to the large scale properties of the universe (besides its acceleration) but not vice versa. The standard model (and any other model too) provides only some approximation to the reality and a slightly modified approximation, which also fits the observations with a satisfactory accuracy, would lead to a gravity theory different from general relativity. It has recently been found that an attempt to reconstruct the $\Lambda$ CDM cosmological model as a $L=f(R)$ gravity provides the required $L=R-2 \Lambda$ as one of a number of solutions but not as the one most probable (i.e. it is not the solution corresponding to attractor points of an autonomous system) [29]. In short: from an approximate solution it is impossible to reconstruct in a reliable way the correct equation of motion. 
On the other hand if the exact form of the spacetime metric accurately corresponding to the distribution of the cosmic matter were known, the problem of how to reconstruct the underlying Lagrangian would be open: the method developed in [28] is specific to $\mathrm{R}-\mathrm{W}$ spacetime and does not work in other cases. We stress that, contrary to a common belief, the $\mathrm{R}-\mathrm{W}$ spacetime is particularly deceptive and unsuitable for recontructing the underlying Lagrangian. This spacetime has a high symmetry and is 'flexible' in the sense that it contains an arbitrary function, so that it is a solution in any metric gravity theory (while Minkowski space is not). Hence for fundamental rather than technical reasons it should not be used for the reconstruction. If one believes at all that it is possible to recover the Lagrangian from one (empirically found) solution, one should apply a solution which does not appear as such in most of gravity theories and is a characteristic feature of a possibly narrow class of theories.

A direct comparison of predictions of a given theory with observations is obstructed by the fact that any nonlinear gravity theory may be formulated in infinite number of distinct frames and many of them have advantage over the original Jordan frame (in which all the theories are initially formulated) in displaying the number of degrees of freedom, the particle spectrum and the dynamics of these fields. Since the problem of which frame is physical (i.e. consists of directly measurable dynamical variables) still remains a matter of a vivid debate and since for this reason any agreement (or disagreement) of the given theory with the observational data may be criticized, instead of attempting to deduce a gravity theory from the data and prior to attempting such a confrontation for a chosen theory, one should verify if the theory meets the general requirements imposed on a classical field theory. A general $L=f\left(g_{\mu \nu}, R_{\alpha \beta \mu \nu}\right)$ metric theory has eight degrees of freedom and describes a gravitational triplet consisting of the metric, a massive spin-two field and a massive scalar field. For $L=f(R)$ the spin-2 field disappears. All the $L=f\left(g_{\mu \nu}, R_{\alpha \beta}\right)$ theories less differ from each other than it is expected in the original Jordan frame since they may be mapped onto general relativity (including the two massive fields) and in this sense the latter theory is clearly distinguished as being a universal Hamiltonian image of all these theories.

How many conditions a gravity theory should satisfy to be regarded a viable one may be disputable (e.g. should it have quantization properties better than general relativity?). The criterion that a theory have a stable ground state being a maximally symmetric spacetime is indisputable. This criterion is investigated in the subsequent Paper II. It is shown there that there is an infinity of viable gravity theories. One should therefore apply other viability criteria coming from classical field theory. The most natural one would be the existence of a proper Newtonian limit. However at present it is difficult to formulate this condition in a precise and effective way. And we stress that at the present level of knowledge it is impossible to find out a system of selection rules allowing one to choose a unique theory out of the whole set of the metric gravity theories. Therefore 
above all one should provide a deeper physical motivation (different from mere applying a number of selection rules and from the wish to account for the cosmic acceleration) for choosing a specific Lagrangian rather than any other. In other terms the cosmic acceleration should be a new important test for a modified gravity theory but it does not provide a way for reconstructing it.

\section{Acknowledgments}

I am grateful to Michael Anderson, Piotr Bizoń, Piotr Chruściel, Helmut Friedrich, Zdzisław Golda and Barton Zwiebach for extensive discussions, helpful comments and explanations. This work is supported in part by a Jagellonian University grant.

\section{References}

[1] K. S. Stelle, Phys. Rev. D16 (1977) 953.

[2] A. A. Starobinsky, Phys. Lett. 91B (1980) 99; R. Kerner, Gen. Rel. Grav. 14 (1982) 453; A. A. Starobinsky and H.-J. Schmidt, Class. Quantum Grav. 4 (1987) 695.

[3] S. M. Carroll, V. Duvvuri, M. Trodden and M. S. Turner, Phys. Rev. D70 (2004) 043528.

[4] S. M. Carroll, A. De Felice, V. Duvvuri, D. A. Easson, M. Trodden and M. S. Turner, Phys. Rev. D71 (2005) 063513.

[5] S. Nojiri and S. D. Odintsov, Phys. Rev. D68 (2003) 123512.

[6] S. Nojiri and S. D. Odintsov, Phys. Rev. D74 (2006) 086005 hep-th/0608008.

[7] S. Capozziello, A. Stabile and A. Troisi, Mod. Phys. Lett. A21 (2006) 2291 gr-qc/0603071.

[8] J. A. R. Cembranos, Phys. Rev. D73 (2006) 064029.

[9] A. W. Brookfield, C. van de Bruck and L. M. H. Hall, Phys. Rev. D74 (2006) 064028, hep-th/0608015.

[10] A. D. Rendall, Class. Quantum Grav. 24 (2007) 667, gr-qc/0611088; Class. Quantum Grav. 21 (2004) 2445.

[11] T. Multamäki and I. Vilja, Phys. Rev. D74 (2006) 064022, astro-ph/0606373. 
[12] A. L. Erickcek, T. L. Smith and M. Kamionkowski, Phys. Rev. D74 (2006) 121501, astro-ph/0610483.

[13] V. Kagramanova, J. Kunz and C. Lämmerzahl, Phys. Lett. B634 (2006) 465.

[14] G. J. Olmo, Phys. Rev. Lett. 95 (2005) 261102; Phys. Rev. D72 (2005) 083505; Phys. Rev. D75 (2007) 023511, |gr-qc/0612047].

[15] M. L. Ruggiero and L. Iorio, JCAP 0701 (2007) 010, gr-qc/0607093.

[16] L. Amendola, D. Polarski and S. Tsujikawa, Phys. Rev. Lett. 98: 131302, 2007.

[17] T. Chiba, Phys. Lett. B575 (2003) 1.

[18] T. Chiba, T. L. Smith and A. L. Erickcek, astro-ph/0611867.

[19] T. Clifton and J. D. Barrow, Phys. Rev. D72 (2005) 103005.

[20] I. Navarro and K. van Acoleyen, JCAP 0702: 022, 2007 gr-qc/0611127.

[21] S. Das, N. Banerjee and N. Dadhich, Class. Quantum Grav. 23 (2006) 4159.

[22] A. de la Cruz-Dombriz and A. Dobado, Phys. Rev. D74 (2006) 087501, gr-qc/0607118.

[23] D. A. Easson, JCAP 0702: 004, 2007 astro-ph/0608034.

[24] R. P. Woodard, Proceedings of 3rd Aegean Summer School "The Invisible Universe: Dark Matter and Dark Energy", September 2005, astro-ph/0601672.

[25] R. Bean, D. Bernat, L. Pogosian, A. Silvestri and M. Trodden, Phys. Rev. D75 (2007) 064020.

[26] O. Mena, J. Santiago and J. Weller, Phys. Rev. Lett. 96 (2006) 041103.

[27] M. Amarzguioui, O. Elgaroy, D. F. Mota and T. Multamäki, Astron. Astrophys. 454 (2006) 707, astro-ph/0510519.

[28] S. Capozziello, V. F. Cardone and A. Troisi, Phys. Rev. D71 (2005) 043503.

[29] S. Fay, S. Nesseris and L. Perivolaropoulos, gr-qc/0703006].

[30] R. Dick, Gen. Rel. Grav. 36 (2004) 217.

[31] A. J. Bustelo and D. E. Barraco, Class. Quantum Grav. 24 (2007) 2333 |gr-qc/0611149. 
[32] D. G. Boulware and S. Deser, Phys. Rev. D6 (1972) 3368.

[33] E. S. Fradkin and A. A. Tseytlin, Nucl. Phys. B201 (1982) 469.

[34] N. Barth and S. M. Christensen, Phys. Rev. D28 (1983) 1876.

[35] A. Hindawi, B. A. Ovrut and D. Waldram, Phys. Rev. D53 (1996) 5597.

[36] R. K. Sachs, Gravitational radiation, in: Relativity, Groups and Topology, Les Houches 1963, edited by C. DeWitt and B. DeWitt, Gordon and Breach, New York 1964, pp. 523-562.

[37] G. Magnano and L. M. Sokołowski, Phys. Rev. D50 (1994) 5039, gr-qc/9312008].

[38] G. Magnano, M. Ferraris and M. Francaviglia, Gen. Rel. Grav. 19 (1987) 465.

[39] A. Jakubiec and J. Kijowski, Phys. Rev. D37 (1988) 1406; Gen. Rel. Grav. 19 (1987) 719 .

[40] G. Magnano, M. Ferraris and M. Francaviglia, Class. Quantum Grav. 7 (1990) 557.

[41] G. Magnano and L .M. Sokołowski, Ann. Phys. (N.Y.) 306 (2003) 1.

[42] L. M. Sokołowski and A. Staruszkiewicz, Class. Quantum Grav. 23 (2006) 5907.

[43] K. S. Stelle, Gen. Rel. Grav. 9 (1978) 353.

[44] J.C. Alonso, F. Barbero, J. Julve and A. Tiemblo, Class. Quantum Grav. 11 (1994) 865.

[45] A. Hindawi, B. A. Ovrut and D. Waldram, Phys. Rev. D53 (1996) 5583.

[46] C. Aragone and S. Deser, Nuovo Cim. 3A (1971) 709; Nuovo Cim. 57B (1980) 33.

[47] E. T. Tomboulis, Phys. Lett. B389 (1996) 225.

[48] R. M. Wald, Class. Quantum Grav. 4 (1987) 1279.

[49] T. Chiba, JCAP 0503 (2005) 008.

[50] M. Gasperini and G. Veneziano, Phys. Reports 373 (2003) 1; E. Alvarez and J. Conde, Mod. Phys. Lett. A17 (2002) 413.

[51] J. Koga and K. Maeda, Phys. Rev. D58 (1998) 064020. 
[52] S. Capozziello, E. de Ritis and A. A. Marino, Class. Quantum Grav. 14 (1997) 3243; R. Dick, Gen. Rel. Grav. 30 (1998) 435; R. Casadio and B. Harms, Mod. Phys. Lett. A14 (1999) 1089; V. Faraoni and E. Gunzig, Int. J. Theor. Phys. 38 (1999) 217; V. Faraoni, E. Gunzig and P. Nardone, Fund. Cosmic Phys. 20 (1999) 121; A. Macías and A. García, Gen. Rel. Grav. 33 (2001) 889; D. N. Vollick, Class. Quantum Grav. 21 (2004) 3813; E. E. Flanagan, Class. Quantum Grav. 21 (2004) 3817.

[53] T. Jacobson, G. Kang and R. C. Myers, Phys. Rev. D52 (1995) 3518.

[54] S. Capozziello, S. Nojiri, S. D. Odintsov and A. Troisi, Phys. Lett. B639 (2006) 135. 